\title{
El envejecimiento de las pastas de cemento reforzadas con fibras de vidrio
}

\author{
M. L. SÁNCHEZ PARADELA y A. DEL AGUILA \\ Departamento de Construcción y Tecnología Arquitectónicas \\ Escuela Técnica Superior de Arquitectura \\ Universidad Politécnica de Madrid. ESPAÑA
}

Fecha de recepción: 20-IV-92

\begin{abstract}
RESUMEN
Las pastas de cemento reforzadas con fibras de vidrio, denominadas popularmente GRC ("Glassfibre Reinforced Cements"), son materiales compuestos que se utilizan ampliamente en el campo de la Construcción desde hace unos 30 años. Sus buenas propiedades mecánicas, ligereza y resistencia a la corrosión y al fuego les hacen idóneos en el proceso de sustitución de los fibrocementos de base amianto en numerosas aplicaciones. Por tal motivo, su empleo se ha extendido en los últimos años, utilizándose fundamentalmente para fabricar paneles de cierre de fachadas y decoración de interiores y en menor grado para tuberías, cubiertas, encofrados permanentes e incluso para "fingers" o brazos de acceso a los aviones en aeropuertos $(1,2,3,4,5)$.
\end{abstract}

Sin embargo, es un hecho conocido desde hace más de 25 años que el GRC sufre un deterioro con el tiempo, que afecta a sus propiedades mecánicas y muy especialmente a su tenacidad, volviéndose frágil y agrietándose bajo pequeñas deformaciones. Las investigaciones realizadas desde entonces para determinar las causas del fenómeno y buscar posibles soluciones al mismo han sido numerosísimas y sería imposible detallarlas aqui por razones de espacio. Trataremos no obstante de resumir los aspectos más relevantes del problema y las soluciones más extendidas hasta el momento.

\begin{abstract}
$S U M M A R Y$
Cement pastes reinforced with glassfibre, popularly called GRC ("Glassfibre Reinforced Cements") are compound materials which have been widely used in the construction field for the last 30 years. Their good mechanical properties, lightness and resistence to corrosion and fire make them siutable in the process of replacing the fibrecements with asbestos base in numerous applications. For that reason, their use has been extended in recent years and they have mainly been used to manufacture panels for façades and interior decoration as well as, to a smaller extent, for plumbing installations, roofs, lost formwork and even for "fingers" at the airports $(1,2,3,4,5)$.
\end{abstract}

Nevertheless, it has been known for over 25 years that GRC suffer deterioration with the time which affects their mechanical properties and especially their tenacity; they become fragile and crack under small deformations. The research carried out to determine the causes of the fenomenon and look for possible solutions has been intense and it would be impossible to give more details here due to the lack of space. We will try, nevertheless, to summarize some more relevant aspects of the problem and the solutions that have reached the highest point of development so far.

\section{LOS MECANISMOS DEL ENVEJECIMIENTO DEL GRC}

Prácticamente desde el comienzo de la utilización del GRC en el campo de la construcción, se comprobó empíricamente que las fibras de vidrio sufrían una degradación de propiedades debida al ataque químico por los álcalis del cemento $(6,7,8)$. Este hecho motivó la proscripción de la fibra $E$ para el refuerzo de los cementos y el desarrollo de fibras de vidrio AR ("alkali resistant") resistentes a los álcalis. La primera generación de fibras $A R$, denominadas comercialmente CemFIL 1, fue desarrollada por Majumdar y se basó esencialmente en la adición de un $16 \%$ aproximadamente de óxido de circonio en la composición del vidrio $(6,7)$. Numerosas investigaciones demostraron que las fibras AR presentaban una capacidad de resistencia al ataque químico de los álcalis muy superior al de las fibras $E$, lo que les hacía idóneas para el refuerzo de las pastas de cemento $(9,10)$. 
No obstante, a pesar de la mayor resistencia de las fibras $A R$ al ataque de los álcalis comparadas con la fibras E, el GRC fabricado con fibras AR siguió mostrando un problema de envejecimiento, reduciéndose su tenacidad y resistencia a impacto con el tiempo hasta valores alarmantemente bajos, especialmente para almacenamiento en agua (11, $12,13)$. Con la nueva generación de fibras $A R$, denominada comercialmente CemFIL2, el GRC muestra un comportamiento con el tiempo muy superior al reforzado con la primera generación de fibras AR, si bien el problema, aunque reducido, dista mucho de estar totalmente resuelto (14).

El deterioro con el tiempo del GRC utilizando fibras AR no puede explicarse por tanto simplemente a partir del ataque de los álcalis a las fibras. Bentur propone la coexistencia de dos mecanismos de degradación del material compuesto, que tendrían mayor o menor importancia según el tipo de fibras y de matriz (15):

- Ataque químico de las fibras de vidrio.

- Crecimiento de productos de la hidratación del cemento entre los filamentos de las fibras.

El primer mecanismo, principal responsable de la degradación de las fibras $E$, sigue subsistiendo aunque con menor importancia cuando se utilizan fibras AR, en cuyo caso el mecanismo predominante pasa a ser el desarrollo y crecimiento de productos de la hidratación del cemento, fundamentalmente cristales de hidróxido de calcio, que rodean los filamentos de vidrio, aumentando la adherencia fibra-matriz y fragilizando el material.

\section{PROCEDIMIENTOS PARA MEJORAR LA DU- RABILIDAD DEL GRC}

Por todo lo indicado en el apartado precedente, no puede aún afirmarse que el problema del envejecimiento del GRC esté totalmente resuelto con la utilización de fibras AR. Se precisan por tanto medidas conducentes a la mejora de la durabilidad del material.

Los distintos procedimientos de reducción de la fragilidad a largo plazo del GRC se pueden agrupar en dos grandes áreas:

a) Utilización de fibras de vidrio con mayor resistencia al ataque de los álcalis.

b) Modificación de la matriz, bien sea con aditivos o utilizando cementos de baja alcalinidad.

Entre los primeros se puede citar el desarrollo de fibras de vidrio con composiciones químicas más resistentes a los álcalis que las de la fibra AR original CemFIL $(16,17,18)$, así como la utilización de tratamientos superficiales que aumenten la resistencia de la fibra al ataque de los álcalis y prevengan la formación y crecimiento de productos de hidratación. Entre estos procedimientos, el desarrollo de la fibra CemFIL 2 es quizás el ejemplo más significativo (19). En este caso el tratamiento superficial de la fibra incluye un inhibidor que se libera gradualmente con el tiempo en el ambiente alcalino de la matriz de cemento y reduce la velocidad de la reacción álcali/vidrio.

En el segundo grupo se incluyen procedimientos de reducción de la agresividad de la matriz, bien sea por adiciones de puzolanas, cenizas volantes o humo de sílice. La adición de puzolanas no evita la pérdida de resistencia y tenacidad del GRC, pero reduce la velocidad de fenómeno $(19,20,21)$. Los mejores resultados se alcanzan con un contenido del $40 \%$ de cenizas volantes (22). Bentur estudió distintos procedimientos de incorporar humo de sílice, llegando a la conclusión de que la inmersión de los hilos de vidrio en una pasta de humo de sílice antes de su incorporación a la matriz de cemento es el método más eficaz para la mejora de la durabilidad del GRC (23).

Dentro de los procedimientos de modificación de la matriz, debemos citar también la utilización de cementos especiales, con menor $\mathrm{pH}$ que el cemento Portland ordinario. Se ha investigado la utilización de cementos aluminosos (24) y siderúrgicos $(25,26)$, con resultados desiguales. Recientemente, se ha desarrollado en Japón un nuevo cemento especial de baja alcalinidad para su empleo como matriz en el GRC $(17,27,28)$; los resultados de ensayos de envejecimiento acelerado muestran un excelente comportamiento del GRC fabricado con dicho cemento.

Finalmente, indicaremos que, dentro de los métodos de mejora de la durabilidad del GRC, se ha desarrollado recientemente un nuevo proceso, basado en la adición a la matriz de cemento de un producto que reduce la cal libre $(29,30)$. El producto, denominado comercialmente Metakaolin, es una puzolana sintética, obtenida por calentamiento de caolinita en aire entre 700 y $900^{\circ} \mathrm{C}$. Los resultados de ensayos de flexión de GRC con adiciones de este producto muestran un comportamiento a largo plazo muy superior al obtenido con cemento Portland ordinario sin aditivos.

En resumen, podríamos concluir este apartado afirmando que en los últimos años se han propuesto numerosos procedimientos de mejora de la durabilidad del GRC con resultados dispares y que, en la mayoría de los casos, no han llegado a industrializarse por razones fundamentalmente económicas, por lo que aún existe campo abierto de investigación para analizar soluciones alternativas.

\section{PROGRAMA EXPERIMENTAL}

Con el fin de analizar posibles mejoras de la durabi- 
lidad del GRC mediante aditivos a la matriz de cemento, se ha llevado a cabo un amplio programa experimental, estudiando distintas mezclas. Parte del trabajo realizado ya ha sido publicado anteriormente (31) y no se repetirá aquí. Simplemente, indicaremos que como conclusiones de dicho trabajo se puede afirmar que la adición de un polímero termoplástico "Forton" a la matriz de cemento no resulta un método eficaz para evitar el envejecimiento del GRC.

En el programa realizado con posterioridad a la referida publicación, se ha analizado el posible efecto beneficioso de dos tipos distintos de aditivos inorgánicos, por un lado el humo de sílice (que en forma abreviada denominaremos SF) que, como se indicó en el apartado anterior, es considerado por muchos autores como un buen candidato para mejorar la durabilidad del GRC y por otro lado un producto inorgánico, exento de cloruros, que se utiliza comúnmente como plastificante y acelerador del fraguado del hormigón y que en lo sucesivo denominaremos de forma abreviada como aditivo $A$.

\subsection{Fabricación de probetas}

Se han realizado ensayos de flexión en 3 puntos sobre probetas de dimensiones aproximadas de $250 \times 50 \times 10 \mathrm{~mm}$, con una distancia entre apoyos de $200 \mathrm{~mm}$. Para la obtención de las probetas, se procedió en primer lugar a fabricar placas de $1 \times 1$ $\mathrm{m}^{2}$ aproximadamente y $10 \mathrm{~mm}$ de espesor.

Se fabricaron 4 placas utilizando moldes de conglomerado de madera a los que se aplicó un desencofrante. En todos los casos se utilizó cemento $1 / 45 \mathrm{~A}$ y fibras de vidrio CemFIL 2 de $36 \mathrm{~mm}$ de longitud. La relación agua/cemento fue de 0,35. La composición de las cuatro placas se indica en la tabla 1. Como puede comprobarse, las placas I y || sirven de control del efecto de los aditivos.

TABLA I

Composición de las mezclas

\begin{tabular}{|l|l|}
\hline PLACA I & $\begin{array}{l}\text { Cemento Portland }+1,5 \% \text { Fibra } \\
\text { de vidrio }\end{array}$ \\
PLACA II & $\begin{array}{l}\text { Cemento Portland }+5 \% \text { Fibra de } \\
\text { vidrio } \\
\text { Cemento Portland }+5 \% \text { Fibra de } \\
\text { vidrio }+1 \% \text { Aditivo A }\end{array}$ \\
PLACA IV & $\begin{array}{l}\text { Cemento Portland }+1,5 \% \text { Fibra } \\
\text { de vidrio }+ \text { SF }\end{array}$ \\
\hline
\end{tabular}

Las placas I, II y III se fabricaron por el método denominado "spray-up" o proyección simultánea de pasta de cemento y fibra de vidrio (foto 1). La pasta se prepara previamente, amasando el cemento con

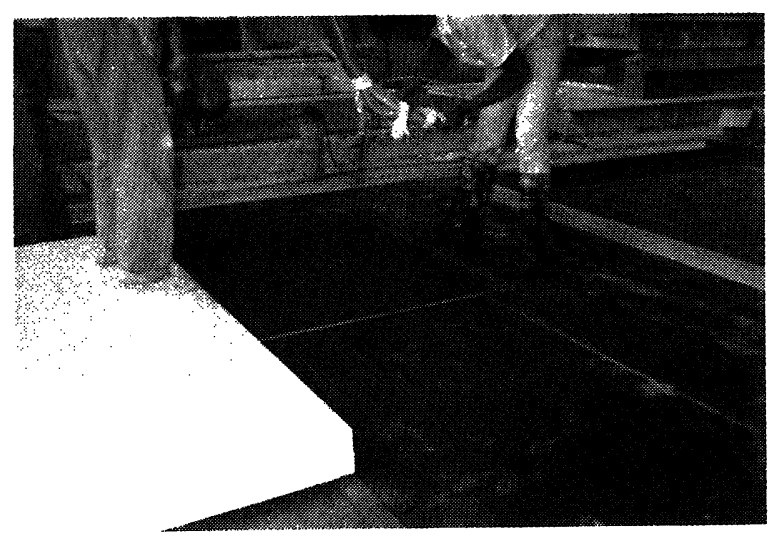

Foto 1.-Fabricación de placas por proyección simultánea.

agua y en su caso con los aditivos en una amasadora, y la máquina proyecta la pasta sobre el molde simultáneamente con la fibra de vidrio cortada de forma automática a la longitud deseada a partir de una bobina. A continuación se alisa la superficie con un rodillo metálico y se finaliza pasando una llana vibrante y llana normal.

La placa IV se fabricó de forma distinta. Por un lado se amasó el cemento con agua, y por otro lado en una cubeta se mezclaron las fibras de vidrio, previamente cortadas, con el humo de sílice y unas gotas de agua para lograr una perfecta impregnación de las fibras. A continuación, en otra cubeta se vertieron ambas mezclas y se amasaron manualmente durante unos minutos hasta lograr una buena dispersión. Finalmente se vertió la mezcla sobre el molde y se finalizó pasando llana vibrante y llana normal.

Las placas se mantuvieron cubiertas con plásticos hasta las 48 horas, en que se desmoldaron y se trasladaron a la cámara húmeda para completar un curado de 28 días a $20^{\circ} \mathrm{C}$ y $95 \%$ de humedad.

Dos días antes del final del curado, las placas se sacaron de la cámara húmeda y se cortaron en probetas de $250 \times 50 \times 10 \mathrm{~mm}$ utilizando una cortadora radial con refrigeración por agua (foto 2 ), obteniéndose por tanto 80 probetas por cada placa, que tras ser marcadas y numeradas se devolvieron a la cámara húmeda.

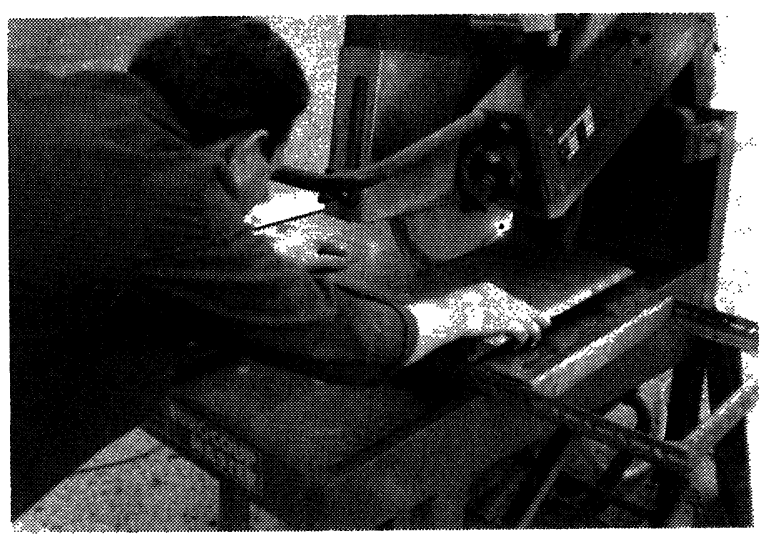

Foto 2.--Corte de probetas. 


\subsection{Ensayos de flexión}

Transcurrido el período de curado, se tomaron 6 probetas de cada mezcla para realizar ensayos de flexión en 3 puntos, además de lo cual se extrajeron al azar conjuntos de 6 probetas de cada mezcla para ser sometidos a envejecimiento acelerado en agua a $50^{\circ} \mathrm{C}$ a distintos tiempos.

Los ensayos de flexión se realizaron en una máquina universal de ensayos de 10 t en capacidad. Se utilizó una velocidad de desplazamiento de carro de $1 \mathrm{~mm} / \mathrm{min}$., que se mantuvo constante en todos los ensayos realizados. (La foto 3 muestra el montaje experimental empleado).

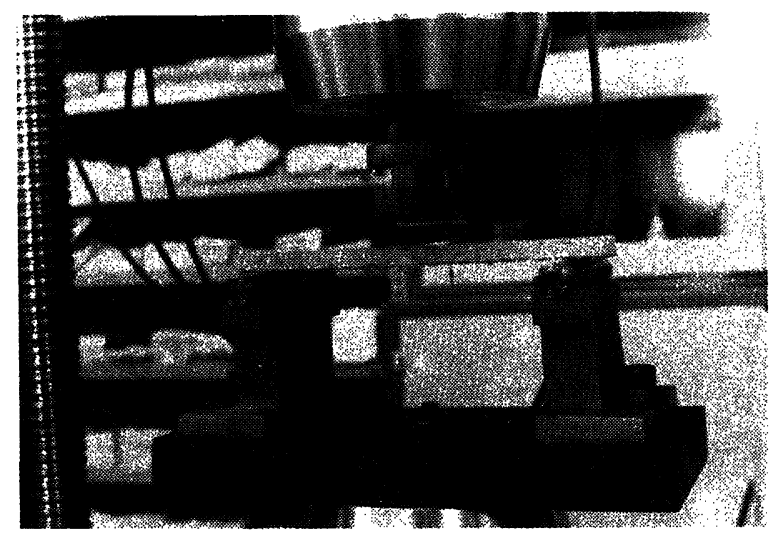

Foto 3.-Dispositivo experimental para los ensayos de flexión.

Las medidas de carga, proporcionadas por la célula de carga de la máquina y de desplazamiento del carro, medidas por un transductor LVDT de la máquina, se recogen en un Sistema de Adquisición de Datos, almacenándose parejas de datos cada décima de segundo. Los resultados se presentan en forma de gráficos carga-desplazamiento, dibujados por el plotter del ordenador.

A partir de los registros carga-desplazamiento, se calculó el límite de proporcionalidad (LOP) y el módulo de rotura (MOR) de cada una de las probetas ensayadas, utilizando la siguiente expresión:

$$
\sigma=\frac{3}{2} \frac{P L}{b c^{2}}
$$

donde:

$\sigma$ es la tensión (LOP o MOR),

$P$ es la carga correspondiente,

L es la distancia entre apoyos $(20 \mathrm{~cm})$,

$b$ es el ancho de la probeta,

$c$ es el espesor de la probeta.

\subsection{Envejecimiento acelerado}

El empleo del método de envejecimiento acelerado se basa en la medida de la pérdida de propiedades mecánicas de probetas almacenadas a temperatu- ras altas (usualmente se utiliza $50^{\circ} \mathrm{C}$ ) en tiempos cortos, como medio de predecir la pérdida de dichas propiedades a temperatura ambiente durante largos períodos de tiempo.

La validez de este método queda establecida cuando los datos de envejecimiento acelerado se correlacionan con datos obtenidos a partir de ensayos de envejecimiento en ambientes naturales. Dicha correlación está bien demostrada para el ambiente de Inglaterra (32), para el cual 1 día de envejecimiento acelerado a $50^{\circ} \mathrm{C}$ equivale a 101 días de envejecimiento natural. Correlaciones de este tipo se han obtenido para diversas localizaciones de distintos países con climas muy dispares. Aún falta por encontrarse la mencionada correlación para España, aunque lo cierto es que habría que obtenerla para distintas regiones, dada la variabilidad del clima en nuestro país.

Así pues, se han sometido a envejecimiento acelerado series de 6 probetas de cada una de las mezclas en baño de agua a $50^{\circ} \mathrm{C}$ durante 1,4 y 8 semanas (equivalentes aproximadamente a 2, 8 y 16 años de envejecimiento natural en Inglaterra), tras lo cual se han realizado ensayos de flexión en 3 puntos idénticos a los realizados con probetas tras el curado de 28 días.

\section{RESULTADOS EXPERIMENTALES}

A modo de ejemplo, las figuras 1 a 4 muestran algunas de las curvas experimentales carga-desplazamiento obtenidas con probetas de cada una de las 4 mezclas a los 28 días y tras 1 semana de envejecimiento acelerado. Aunque los resultados dependen de las dimensiones reales de cada probeta, ya puede adelantarse a la vista de los gráficos que las probetas de la placa I presentan una resistencia menor que las de la placa II, como corresponde a su menor contenido de fibras y asimismo las probetas de la placa IV tienen menor resistencia y tenacidad que las de la placa I. Si recordamos que la placa IV incorpora humo de sílice a la composición de la placa I, hemos de concluir que este aditivo no ha resultado beneficioso a efectos de reducir el problema del envejecimiento del GRC.

Por el contrario, las probetas de la placa III presentan niveles de resistencia más altos que las correspondientes de la placa II, por lo que se puede concluir que el aditivo $A$, que es eficaz para aumentar la resistencia del GRC a corto plazo, se muestra asimismo beneficioso para mantener niveles de resistencia más altos en probetas envejecidas.

Este resultado se comprueba más claramente observando la tabla 2 , en la que se recogen de forma resumida los valores medios de LOP, MOR y desplazamiento de rotura de las cuatro mezclas a las distintas edades ensayadas. Puede comprobarse que incluso para tiempos de envejecimiento 


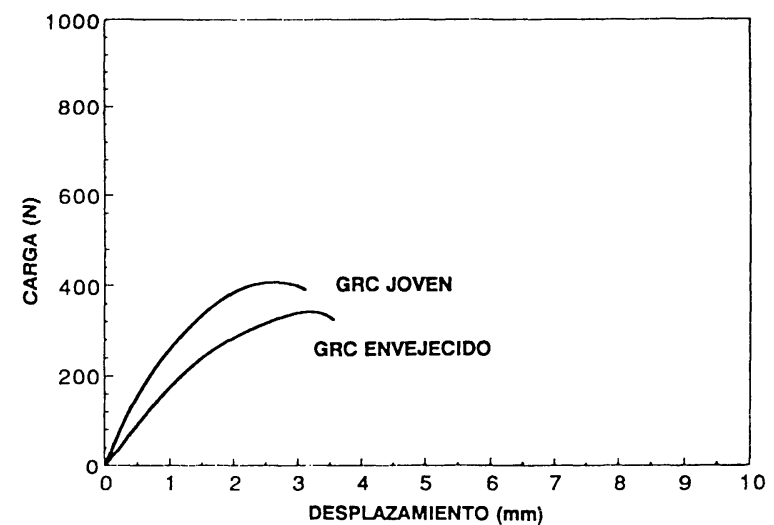

Fig. 1.-Curvas carga-desplazamiento en flexión para probetas de la placa I. GRC joven (28 días) y GRC envejecido 1 semana.

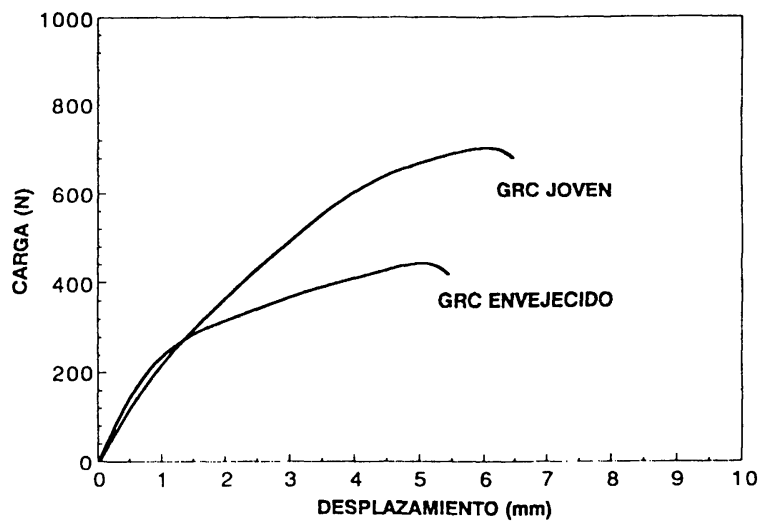

Fig. 2.-Curvas carga-desplazamiento en flexión para probetas de la placa II. GRC joven (28 días) y GRC envejecido 1 semana.

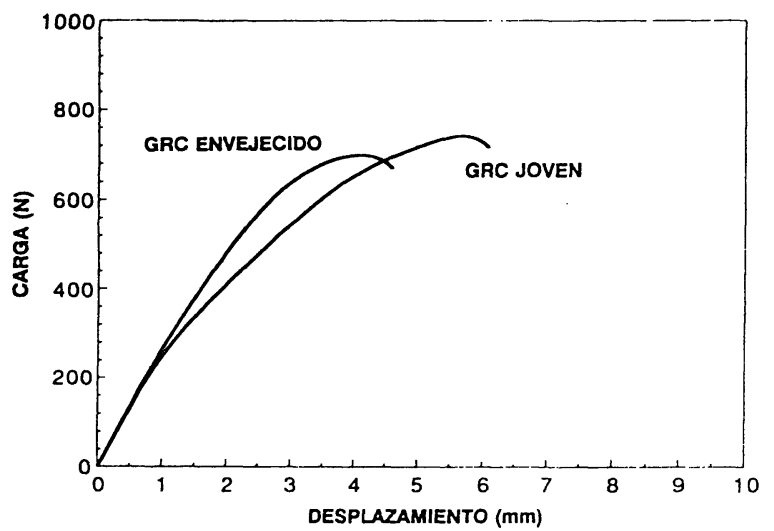

Fig. 3.-Curvas carga-desplazamiento en flexión para probetas de la placa III. GRC joven (28 dias) y GRC envejecido 1 semana.

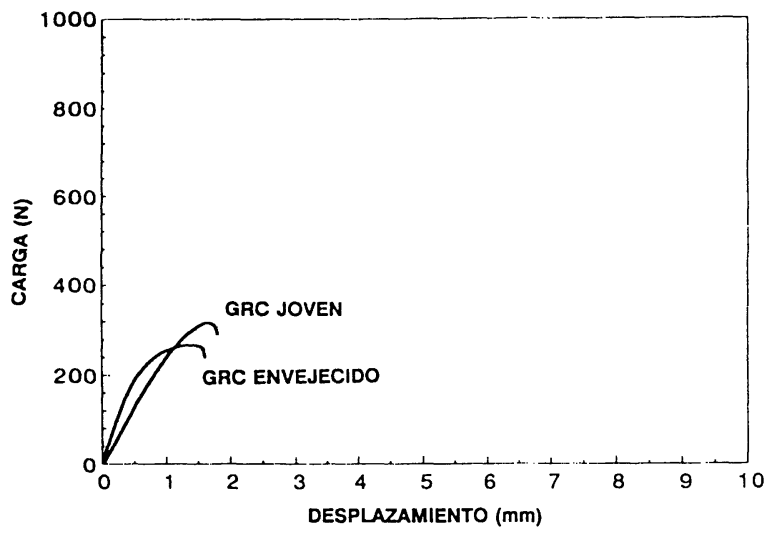

Fig. 4.-Curvas carga-desplazamiento en flexión para probetas de la placa IV. GRC joven (28 días) y GRC envejecido 1 semana.

TABLA 2

Resultados experimentales (valores medios)

\begin{tabular}{|c|c|c|c|c|c|}
\hline \multirow{2}{*}{ Placa } & \multirow{2}{*}{ Propiedad } & \multirow{2}{*}{ GRC joven } & \multicolumn{3}{|c|}{ GRC envejecido $\left(50^{\circ} \mathrm{C}\right)$} \\
\hline & & & 1 semana & 4 semanas & 8 semanas \\
\hline \multirow{3}{*}{1} & LOP (MPa) & 7,5 & 7,3 & 5,9 & 6,5 \\
\hline & MOR (MPa) & 13,7 & 11,9 & 12,0 & 11,8 \\
\hline & $\mathrm{V}_{\max }(\mathrm{mm})$ & 3,1 & 4,0 & 3,6 & 3,8 \\
\hline \multirow{3}{*}{ II } & LOP (MPa) & 8,7 & 9,2 & 9,4 & 9,2 \\
\hline & MOR (MPa) & 18,5 & 14,6 & 14,2 & 15,7 \\
\hline & $V_{\max }(m m)$ & 4,5 & 5,9 & 4,5 & 5,7 \\
\hline \multirow{3}{*}{ III } & LOP (MPa) & 6,9 & 6,4 & 7,6 & 7,5 \\
\hline & MOR (MPa) & 22,7 & 16,8 & 17,7 & 18,3 \\
\hline & $V_{\max }(m m)$ & 5,8 & 5,2 & 4,9 & 4,3 \\
\hline \multirow{3}{*}{ IV } & LOP (MPa) & 4,9 & 5,4 & 5,8 & 6,2 \\
\hline & MOR (MPa) & 8,8 & 7,8 & 9,7 & 9,5 \\
\hline & $\mathrm{V}_{\max }(\mathrm{mm})$ & 1,4 & 1,3 & 1,9 & 1,8 \\
\hline
\end{tabular}


de 8 semanas a $50^{\circ} \mathrm{C}$ (equivalentes a 16 años de envejecimiento natural en Inglaterra), las probetas de la placa III, que incorporan el aditivo A, aunque han experimentado una ligera reducción de resistencia frente a los valores obtenidos a 28 días, siguen manteniendo mejores propiedades mecánicas que las de la placa II, de igual composición pero sin aditivos.

\section{CONCLUSIONES}

Las pastas de cemento reforzadas con fibras de vidrio (GRC) son un material compuesto de amplia utilización en el campo de la construcción.

EI GRC presenta un problema de fragilidad con el tiempo, reduciéndose su resistencia mecánica y su tenacidad, debido al ataque de los álcalis a las fibras de vidrio.

Entre los métodos desarrollados para reducir el problema del envejecimiento del GRC, se encuentran por un lado la obtención de nuevas fibras más resistentes al ataque de los álcalis, y por otro lado la reducción de la agresividad de la matriz, por medio de aditivos o utilizando cementos de baja alcalinidad. Todos estos métodos implican encareci- mientos del producto y su eficacia no siempre está garantizada.

En la presente investigación, se ha estudiado el posible efecto beneficioso sobre la durabilidad del GRC de dos aditivos inorgánicos: el humo de sílice (SF) y un plastificante y acelerador del fraguado comercial (aditivo A).

El primero de ellos no ofrece ningún efecto beneficioso, al menos en la forma en que ha sido incorporado a la mezcla en la presente investigación.

Por el contrario, el aditivo A se ha mostrado eficaz para aumentar la resistencia a flexión del GRC, tanto para material joven como para probetas envejecidas artificialmente mediante almacenamiento en agua a $50^{\circ} \mathrm{C}$ durante 8 semanas. Se trata de un producto barato y sencillo de incorporar a la mezcla, por lo que su utilización industrial no revestiría ninguna dificultad.

En los próximos meses se piensa continuar la investigación, estudiando el comportamiento del GRC con este aditivo en envejecimiento natural, estudio necesario para comprobar el efecto beneficioso logrado en envejecimiento acelerado y que permitiría además buscar la correlación envejecimiento natural frente a envejecimiento acelerado que aún no existe en España.

\section{AGRADECIMIENTO}

Los autores agradecen a la Universidad Politécnica de Madrid la ayuda financiera para la realización de esta investigación a través de la Acción Concertada A91 0020 0201. También quieren expresar su agradecimiento a las empresas PREINCO por la fabricación de las placas y TEXSA por el suministro de los aditivos.

\section{REFERENCIAS}

(1) YOUNG, J.: "Desingning with GRC". Architectural Press. London. 1978.

(2) TRUE, G.: "GRC Production and Uses". Palladian Publications Ltd. London. 1986.

(3) Glassfibre Reinforced Cement Association. "International Survey of GRC Use and Development 1977/87". GRCA. Newport. 1989.

(4) MAJUMDAR, A. J. y LAWS, V.: "Glass Fibre Reinforced Cement". BSP Professional Books. Oxford. 1991.

(5) RODRIGUEZ, J. y JORDAN, M.: "The Use of GRC in Spain and Algeria". Third Int. Symposium on Developments in Fibre Reinforced Cement and Concrete. Sheffield. Julio. 1986.

(6) MAJUMDAR, A. J. y RYDER, J. F.: "Glass Fibre Reinforcement for Cement Products". Glass Technology, Vol. 9, 1986, pp. 78-84.

(7) MAJUMDAR, A. J.: "Glassfibre Reinforced Cement and Gypsum Products". Proc. Roy. Soc. Lond. A, Vol. 319, 1970, pp. 69-78.

(8) BIRYUKOVICH, K. L., BIRYUKOVICH, Y. L. y BIRYUKOVICH, D. L.: "Glassfibre Reinforced Cement". Budivelnik, Kiev, 1964.

(9) MAJUMDAR, A. J.: "Properties of GRC". Fibrous Concrete. Proc. Symp. The Concrete Society. London. 1980, pp. 48-68.

(10) FRANKE, L. y OVERBECK, E.: "Loss in Strength and Damage to Glass Fibres in Alkaline Solutions and Cement Extracts". Durab. Bldg. Mat., Vol. 5, 1987, pp. 73-79. 
(11) MAJUMDAR, A. J., SINGH, B., LANGLEY, A. A. y ALI, M. A.: "The Durability of Glassfibre Cement. The Effect of Fibre Length and Content". J. Mat. Sci., Vol. 15, 1980, pp. 1085-1096.

(12) SINGH, B. y MAJUMDAR, A. J.: "The Effect of Fibre Length and Content on the Durability of Glass Reinforced Cement. Ten Year Results". J. Mat. Sci. Letters, Vol. 4, 1985, pp. 967-971.

(13) ANON: "Properties of GRC: Ten Year Results". Building Research Establishment. Information Paper IP 36/79 BRE. 1979.

(14) BENTUR, A., BEN BASSAT, M. y SCHNEIDER, D.: "Durability of Glassfiber Reinforced Cements with Different Alkali Resistant Glass Fibers". J. Amer. Ceram. Soc., Vol. 68, 1985, pp. 203-208.

(15) BENTUR, A. y MINDESS, S. "Fibre Reinforced Cementitious Composites". Elsevier Applied Science. 1990.

(16) FYLES, K., LITHERLAND, K. L. y PROCTOR, B. A.: "The Effect of Glasfibre Compositions on the Strength Retention of GRC". Third RILEM Int. Symposium on Developments in Fibre Reinforced Cement and Concrete. Sheffield. Julio 1986.

(17) HAYASHI, M., SATO, S. y FUJII, H.: "Some Ways to Improve Durability of GFRC". Proc. Durability of Glass Fibre Reinforced Concrete Symposium. Prestressed Concrete Institute. Chicago, 1985, pp. 270-284.

(18) OHTA, H., YANAGISAWA, O., MUKAIYAMA, T., OHIGASHI, T. y TAKEDA, R.: "New AR Gass Fibre, AR Fibre-Super". Proc. Int. GRCA Congress, Darmstadt, Oct. 1985. GRCA. Newport, pp. 37-43.

(19) PROCTOR, B. A., OAKLEY, D. R. y LITHERLAND, K. L.: "Developments in the Assessment and Performance of GRC over 10 Years". Composites, Vol. 13, 1982, pp. 173-179.

(20) LEONARD, S. y BENTUR, A.: "Improvement of the Durability of Glassfiber Reinforced Cement Using Blended Cement Matrix". Cem. Concr. Res., Vol. 14, 1984, pp. 717-728.

(21) SINGH, B. y MAJUMDAR, A. J.: "Properties of GRC Containing Inorganic Fillers". Int. J. Cem. Comp. and Lightweight Concr., Vol. 3, 1981, pp. 93-102.

(22) SINGH, B. y MAJUMDAR, A. J.: "The Effect of PFA Addition on the Properties of GRC". Int. J. Cem. Comp. and Lightweight Concr., Vol. 7, 1985, pp. 3-10.

(23) BENTUR, A. y DIAMOND, S.: "Effects of Direct Incorporation of Microsilica into GFRC Composites on Retention of Mechanical Properties after Ageing". Proc. Durability of Glass Fibre Reinforced Concrete Symposium. Prestressed Concrete Institute. Chicago, 1985, pp. 337-351.

(24) MAJUMDAR, A. J., SINGH, B. y ALI, M. A.: "Properties of High-Alumina Cement Reinforced with Alkali Resistant Glassfibres". J. Mat. Sci., Vol. 16, 1981, pp. 2597-2607.

(25) MAJUMDAR, A. J., SINGH, B. y EVANS, T. J. "Glass Fibre Reinforced Supersulphated Cement". Composites, Vol. 12, 1981, pp. 171-183.

(26) SINGH, B. y MAJUMDAR, A. J.: "GRC Made from Supersulphated Cement: 10 Year Results". Composites, Vol. 18, 1987, pp. 329-333.

(27) TANAKA, M. y UCHIDA, I.: "Durability of GFRC with Calcium Silicate-C A $_{3}$ S-CS-Slag Type Low Alkaline Cement". Proc. Durability of Glass Fibre Reinforced Concrete Symposium. Prestressed Concrete Institute. Chicago. 1985, pp. 305-314.

(28) AKIHAMA, S., SUENAGA, T., TANAKA, M. y HAYASHI, M.: "Properties of GRC with Low Alkaline Cement". Fiber Reinforced Concrete, Properties and Applications. ACI SP-105. American Concrete Institute. 1987, pp. 189-209.

(29) AMBROISE, J., DEJEAN, J., FOUMBI, J. y PERA, J. "Metakaoline Blended Cements: An Efficient Way to Improve GRC Durability and Ductility". Proc. 6th Int. GRCA Congress. Edinburgh. Oct. 1987. GRCA. Newport, pp. 19-24.

(30) THIERY, J. y GENIS, A.: "High Durability Glass Cement Composites: New Vetrotex System". Proc. 7th Int. GRCA Congress. Maastricht. Sept. 1989. GRCA. Newport, pp. 335-344.

(31) SANCHEZ PARADELA, M. L. y SANCHEZ GALVEZ, V.: "Comportamiento a tracción de cementos reforzados con fibra de vidrio". Informes de la Construcción, Vol. 43, No. 413, 1991, pp. 77-89.

(32) LITHERLAND, K. L., OAKLEY, D. R. y PROCTOR, B. A.: "The Use of Accelerated Ageing Procedures to Predict the Long Term Strength of GRC Composites". Cement and Concrete Research, Vol. 11, 1981, pp. 455-466. 